\title{
An Minimum-Energy-Based High-Degree Polynomial Trajectory Planning and Tracking Control for an LCD Glass-handling Robot
}

\author{
Rong-Fong Fung*, Yi-Hsin Cheng \\ Department of Mechanical and Automation Engineering \\ National Kaohsiung First University of Science and Technology \\ University Road, Yenchau, Kaohsiung City, TaiWan \\ * Corresponding authors' Email: rffung@nkfust.edu.tw
}

\begin{abstract}
A new method of minimum-energy point-to-point (PTP) trajectory planning is proposed for an LCD glass-handing robot, which is driven by a permanent magnet synchronous motor (PMSM). The variable structure controller (VSC) is designed to track the trajectories and compensate LuGre model of frictional torque effects. The robot system is described by a mechanical equation and an electrical equation. To generate the minimum-energy trajectory, we employ a high-degree polynomial with suitable end-point conditions. The real-coded genetic algorithm (RGA) is used to search for the coefficients of the polynomial with the fitness function of minimum-energy input. Finally, numerical simulations of the minimum-energy inputs are compared for various degrees of polynomials. It is concluded that the proposed methodology can effectively design the minimum-energy trajectory, and the nonlinear VSC can track the designed trajectories for the robot system driven by a PMSM.
\end{abstract}

Keywords: LCD glass-handling robot; minimum-energy; permanent magnet synchronous motor (PMSM); realcoded genetic algorithm (RGA); trajectory planning

\section{Introduction}

The subject about trajectory planning has been extensively studied. The problem of robot trajectory planning is a very complex task, and plays a crucial role in design and application of robots in task space. The robot trajectory planning can be accomplished through the way of generating and optimizing ruled surfaces under constraints of kinematics, dynamics and control performances. Based on an algorithm which is capable of obtaining a sequence of feasible robot configurations, a path between the given initial and the goal configurations to be reached is obtained [1]-[3]. Biagiotti and Melchiorri [4] developed the trajectory planning of the computation of desired motion pro- files for the actuation systems of automatic machines.

Huang et al. [5] have proposed a novel minimumenergy point-to-point (PTP) trajectory planning method for a motor-toggle servomechanism. In order to generate a robot's trajectory, the genetic algorithm (GA) was developed to search optimal solutions for the trajectories $[6,7]$. The dynamic formulation of an LCD glass-handling robot driven by a PMSM was formulated by Hamilton's principle [8, 9], where the identification and tracking control were also performed for the system. A survey [10] of controlling ac machines for high dynamic performance including different types of machines and converters with their specific control characteristics was performed.

Canudas de Wit et al. [11] proposed a dynamic fric- 
tional model that combines the stiction behavior, i.e., the Dahl effect, with arbitrary steady-state frictional characteristics which include Stribeck effect. Frictional force is a nonlinear behavior and can cause some difficult problems such as static errors, limit cycles and stick-slip in mechanical systems [12]. The variable structure controller (VSC) can enhance the robustness under plant disturbances and uncertainties [13, 14]. One of the most recent topics in the variablestructure system theory is represented by the secondorder sliding-mode control methodology [13]. Lee et al. [14] proposed an integral sliding-mode control method to obtain zero steady-state error under an external step disturbance and to show its robust control with respect to the parametric variations of the input channel.

In this paper, we adopt the RGA to obtain the polynomial coefficients with a fitness function of minimumenergy input for the PTP motion profile of an LCD glass-handling robot. In this paper, the mathematical model includes the electrical and mechanical equations. The VSC is designed to compensate the frictional torque. Various degrees of polynomials with the minimum-energy inputs are compared in numerical simulations. It is found that the proposed methodology can effectively design the minimum-energy trajectory, and the VSC can track the minimum-energy trajectory for the robot system driven by a PMSM.

\section{Dynamic equations of the mechatronic syste}

In order to completely formulate the dynamic equations, we use the electrical and mechanical equations to describe the LCD glass-handling robot driven by a PMSM.

For the electrical equation, we adopt [10] and have

$$
v_{q}=R_{s} i_{q}+\frac{d}{d t} \lambda_{q}+\omega_{s} \lambda_{d}
$$

where $v_{q}$ is the stator voltage, $R_{s}$ is the stator resistance, $i_{q}$ is the stator current, $\lambda_{q}$ is the stator flux linking, $\omega_{s}$ is the inverter frequency and $\lambda_{d}$ is the stator flux linking.

In Figure 1, the mechatronic system consists of a PMSM, two timing belt drives and three arms. An LCD glass-handling robot is driven with a maximum travel distance $260 \mathrm{~mm}$ in the $X_{1}$ direction by a PMSM. The principle of the motion is that the PMSM drives Arm 1 at point $O_{1}$ to convert into rotation of Arm 1 and then the timing belt 1 curl Gear 1 to pull rotation of Gear 2. At this time, Arm 2 also rotates along with rotation of Gear 2 and then the timing belt 2 curl Gear 4 to pull rotation of Gear 3 .

In order to simplify the dynamic model, we assume that (A) the timing belts have no difference in slipping in the driving and driven gears, (B) the timing belts are rigid (i.e. the stiffness of timing belts are infinite), and (C) the motor shaft and all of the gears and arms are rigid. Here, one degree-of-freedom dynamic model was formulated by Hamilton's principle [8] as follows

$$
\begin{array}{r}
{\left[J_{0}-J_{1} \cos \left(n \phi_{1}\right)+J_{m}\right] \ddot{\phi}_{1}+B_{m} \dot{\phi}_{1}} \\
+\mu \sin \left(n \phi_{1}\right) \dot{\phi}_{1}^{2}=K_{\tau} i_{q},
\end{array}
$$

where

$$
\begin{aligned}
& J_{0}=I_{1}+(n-1)^{2} I_{2}+(1-2 n)^{2} I_{3}+(n-1)^{2} I_{4}+4 I_{5} \\
&+(1-2 n)^{2} I_{6}+m_{2}\left[l_{1}^{2}+(n-1)^{2} b^{2}\right] \\
&+m_{3}\left[l_{1}^{2}+(n-1)^{2} l_{2}^{2}\right]+m_{4} l_{1}^{2}+m_{5} l_{1}^{2} \\
&+m_{6}\left[l_{1}^{2}+(n-1)^{2} l_{2}^{2}\right] \\
& J_{1}=2(n-1)\left[m_{2} b+\left(m_{3}+m_{6}\right) l_{2}\right] l_{1}, \\
& \mu=n(n-1)\left[m_{2} b+\left(m_{3}+m_{6}\right) l_{2}\right] l_{1} .
\end{aligned}
$$

$\phi_{1}, \dot{\phi}_{1}$, and $\ddot{\phi}_{2}$ are the angular displacement, angular speed and angular acceleration, respectively. $J_{m}$ is the moment of inertia of the rotor, $B_{m}$ is the damping coefficient, $K_{t}$ is the motor torque constant of the PMSM, and the detailed notations can also be seen in [8]. Equation (2) is a nonlinear ordinary differential equation, and the coefficients of the moment inertial and the nonlinear damping are functions of the rotor angle.

Two kinematic equations [8] of the geometric constraints in the $X_{1}$ and $Y_{1}$ directions can be described as

$$
\begin{aligned}
& X_{1}=l_{1} \cos \phi_{1}+l_{2} \cos \left(\phi_{1}-n \phi_{1}\right), \\
& Y_{1}=l_{1} \sin \phi_{1}+l_{2} \sin \left(\phi_{1}-n \phi_{1}\right) .
\end{aligned}
$$

where $n$ is the radius ratio of $\gamma_{1} / \gamma_{2}$. Equations (3) and (4) are the relationships between the translations of point $O_{3}$ and rotation of Arm 1 .

\section{Point-to-point trajectory design}

The minimum-energy PTP trajectory [4], [5] is designed on the basis of a high-degree polynomial, which is able to satisfy desired constraints of angular displacement, speed, acceleration and jerk at the start time $t_{0}$ and end time $T$. From a mathematical point of view, the problem is then to find a function such that

$$
\phi_{l}=\phi_{1}^{*}(t), t \in\left[t_{0}, T\right],
$$

where the superscript $*$ indicates the designed trajectory, $\phi_{1}^{*}(t)$ and is the designed angular displacement of Arm 1. 


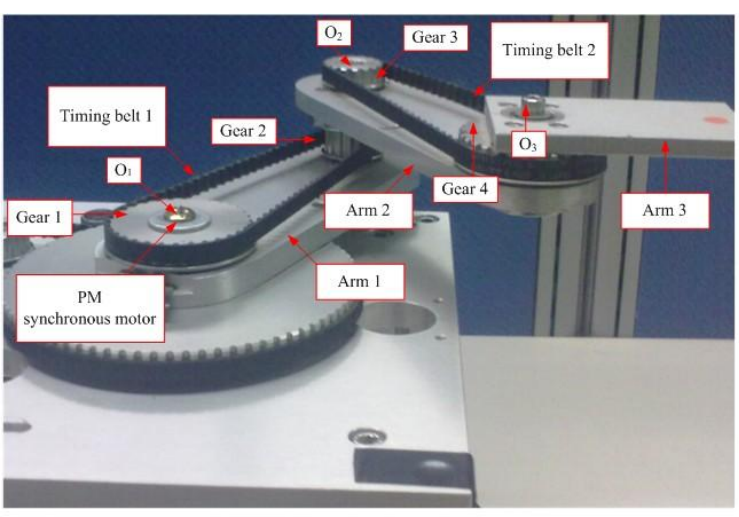

(a)

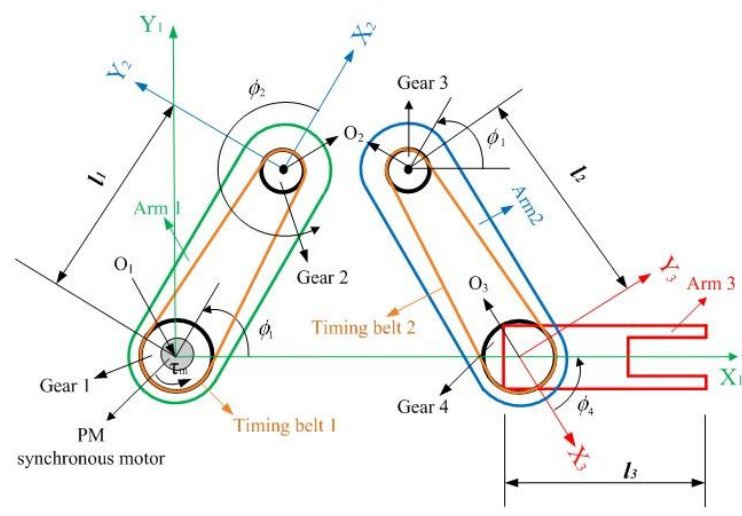

(b)

Figure 1 The LCD glass-handling robot driven by a PMSM. (a) The experimental equipment. (b) The geometrical mode.

\subsection{Polynomial trajectories}

The trajectory planning problem can be easily solved by considering a polynomial function as

$$
\phi_{l}^{*}(t)=a_{0}+a_{1} t+a_{2} t^{2}+a_{3} t^{3}+\cdots+a_{m} t^{m}
$$

where each coefficient $a_{i}, i=0,1, \cdots, m$, is a real number; $a_{m} \neq 0$, and $m$ is a positive integer. There are $m+1$ coefficients to be determined such that the constraints are satisfied at the start and end time. The degree $m$ of the polynomial depends on the number of constraints to be satisfied and on the desired smoothness of the resulting motion.

Considering a polynomial of degree twelve, and the angular displacement, speed, acceleration and jerk are zero at the start time, we have

$$
\begin{aligned}
& \phi_{1}^{*}(0)=a_{0}=0, \\
& \dot{\phi}_{1}^{*}(0)=a_{1}=0, \\
& \ddot{\phi}_{1}^{*}(0)=a_{2}=0, \\
& \dot{\phi}^{*} ł 11(0)=a_{3}=0 .
\end{aligned}
$$

The angular displacement is $\Phi$, and the angular speed, acceleration and jerk are zero at the end time $T$, thus we have

$$
\begin{aligned}
& \phi_{1}^{*}(T)=\Phi, \\
& \dot{\phi}_{1}^{*}(T)=0, \\
& \ddot{\phi}_{1}^{*}(T)=0, \\
& \dot{\phi}_{1}^{*}(T)=0 .
\end{aligned}
$$

where $\Phi$ is the maximum angular displacement. In this paper, we have $\Phi=1.57 \mathrm{rad}$, which is obtained from (3) with the maximum distance $X_{l}=260 \mathrm{~mm}$ in the $X_{1}$ direction of the LCD glass-handling robot. Substituting the eight conditions (7)-(14) into the twelvedegree polynomial (6), we have the four analytic solutions

$$
\begin{gathered}
a_{4}=35 \frac{\Phi}{T^{4}}+T^{4} a_{8}+4 T^{5} a_{9}+10 T^{6} a_{10} \\
+20 T^{7} a_{11}+35 T^{8} a_{12}, \\
a_{5}=-84 \frac{\Phi}{T^{5}}-4 T^{3} a_{8}-15 T^{4} a_{9}-36 T^{5} a_{10} \\
\quad-70 T^{6} a_{11}-120 T^{7} a_{12}, \\
a_{6}=70 \frac{\Phi}{T^{6}}+6 T^{2} a_{8}+20 T^{3} a_{9}+45 T^{4} a_{10} \\
+84 T^{5} a_{11}+140 T^{6} a_{12}, \\
\\
a_{7}=-20 \frac{\Phi}{T^{7}}-4 T a_{8}-10 T^{2} a_{9}-20 T^{3} a_{10} \\
\quad-35 T^{4} a_{11}-56 T^{5} a_{12} .
\end{gathered}
$$

The coefficients $a_{4}-a_{7}$ are to be determined once $a_{8}-a_{12}$ are known. The coefficients $a_{8}-a_{12}$ in (15)(18) for the twelve-degree polynomial trajectory are to be determined by the following RGA method.

\subsection{Input energy for the PTP motion}

The whole PMSM may be considered thermodynamically as an energy converter. It takes electrical input energy from control command and then outputs the mechanical work to drive the Arm. The absolute value of input energy to the PMSM during the whole time $T$ is defined as

$$
E(z)=\int_{0}^{T}\left|i_{q}^{*} v_{q}^{*}\right| d t
$$

where

$$
\begin{aligned}
i_{q}^{*}= & \frac{l}{K_{t}}\left(\left[J_{0}-J_{1} \cos \left(n \phi_{1}^{*}\right)+J_{m}\right] \ddot{\phi}_{1}^{*}+B_{m} \dot{\phi}_{1}^{*}\right. \\
& \left.+\mu \sin \left(n \phi_{1}^{*}\right) \dot{\phi}_{1}^{* 2}\right)
\end{aligned}
$$




$$
v_{q}^{*}=R_{s} i_{q}^{*}+L_{q} \frac{d}{d t} i_{q}^{*}+p \lambda_{d} \dot{\phi}_{l}^{*} .
$$

The $i_{q}^{*}$ is electric current, and can be obtained by the designed trajectory $\phi_{1}^{*}, \dot{\phi}_{1}^{*}$ and $\ddot{\phi}_{1}^{*}$ from (2). The $v_{q}^{*}$ is the control command, and can be obtained by substituting $i_{q}^{*}$ and $\dot{\phi}_{1}^{*}$ into (21). Therefore, it can be shown that the absolute value of input energy in (19) can be obtained once the designed trajectory is obtained.

\section{The real-coded genetic algorithm}

The RGA is an optimization searching algorithm, which simulates evolution mechanism on a computerbased platform in conjunction with natural selection and genetic mechanism. The chromosomes are expressed by vectors and each element of vectors is called a gene. The initial real-valued genes in the chromosomes are gotten through generating a sequence of real-valued variable by a randomly limited range. All chromosomes compose of a population and are evaluated according to the pre-given evaluation index and given fitness values. According to the fitness values, the reproduction, crossover and mutation operations are carried out among or between the chromosomes. The chromosomes having less fitness values are given up, and the chromosomes having larger fitness values are left. Those left chromosomes compose of a new population, which maybe better than the old.

\subsection{Fitness function}

The RGA continuously searches the better chromosomes in this way until the converging index is satisfied. By using the RGA merit, we can obtain the unknown coefficients $a_{8}$ - $a_{12}$ in (15)-(18).

The RGA procedure is shown in Figure 2, where the reproduction procedure adopts the roulette wheel selection, and the crossover and uniform mutation adopt the methods as in $[6,7]$. How to define the fitness function is the key point of the genetic algorithm, since the fitness function is a figure of merit, and could be computed by using any domain knowledge.

In this paper, the fitness function $f(z)$ in the RGA method is defined as

$$
\begin{aligned}
& f(z)=\frac{1}{E(z)} \\
& z=\left[\begin{array}{lllll}
a_{8} & a_{9} & a_{10} & a_{11} & a_{12}
\end{array}\right]
\end{aligned}
$$

where $z$ is a parameter vector of polynomial coefficients, and $E(z)$ is the total absolute input energy.

\subsection{Increasing function}

During the PTP motion profile of the mechanical system, the trajectory for the angular displacement is required to be designed as a monotonously increasing function from the start to the end points. Suppose that $\phi_{1}^{*}(t)$ is the designed PTP motion profile defined during $0 \leq t \leq T$ for $t_{i}<t_{i+1}$, the monotonously increasing function is defined as

$$
\phi_{1}^{*}\left(t_{i}\right)<\phi_{1}^{*}\left(t_{i+1}\right)
$$

where the subscript $i$ stands for the $i^{\text {th }}$ sampling time.

This constraint for a monotonously increasing function is inserted in the procedure of the RGA method as shown in Figure 2. Without using this constraint (24), the identified coefficients of polynomials are not unique in the numerical simulations by the RGA method [5].

\section{Variable Structure Controller Design}

In order to achieve good tracking control with the minimum-energy input (19) even if external disturbances exist, we utilize the VSC to obtain the best control performance in this paper.

\subsection{Frictional model}

To consider the frictional torque, we adopt the $\mathrm{Lu}-$ Gre model [11, 12], which contains a state variable representing the average deflection of elastic bristles, which are visualized on the contacting surfaces. The resulting model faithfully captures the majority of known frictional behaviors, such as the pre-sliding displacement, frictional lag, varying break-away torque and stick-slip motion.

The frictional torque of LuGre model [11] is defined as

$$
\tau_{f}=\sigma_{0} z+\sigma_{1} \frac{d z}{d t}+\sigma_{2} \dot{\phi}_{1}
$$

where $\sigma_{0}$ is the stiffness, $\sigma_{1}$ is a damping and $\sigma_{2}$ is a viscous frictional coefficient. The average deflection of the bristles is denoted by $\mathrm{z}$ and is modeled by

$$
\frac{d z}{d t}=\dot{\phi}_{1}-\frac{\left|\dot{\phi}_{1}\right|}{g\left(\dot{\phi}_{1}\right)} z
$$

where $\dot{\phi}_{1}$ is the relative velocity between the two surfaces. On the right-hand side, the first term gives a deflection that is proportional to the integral of the relative velocity. The second term asserts that the deflection $z$ approaches the value,

$$
z=\frac{\dot{\phi}_{1}}{\left|\dot{\phi}_{1}\right|} g\left(\dot{\phi}_{1}\right)=g\left(\dot{\phi}_{1}\right) \operatorname{sign}\left(\dot{\phi}_{1}\right)
$$




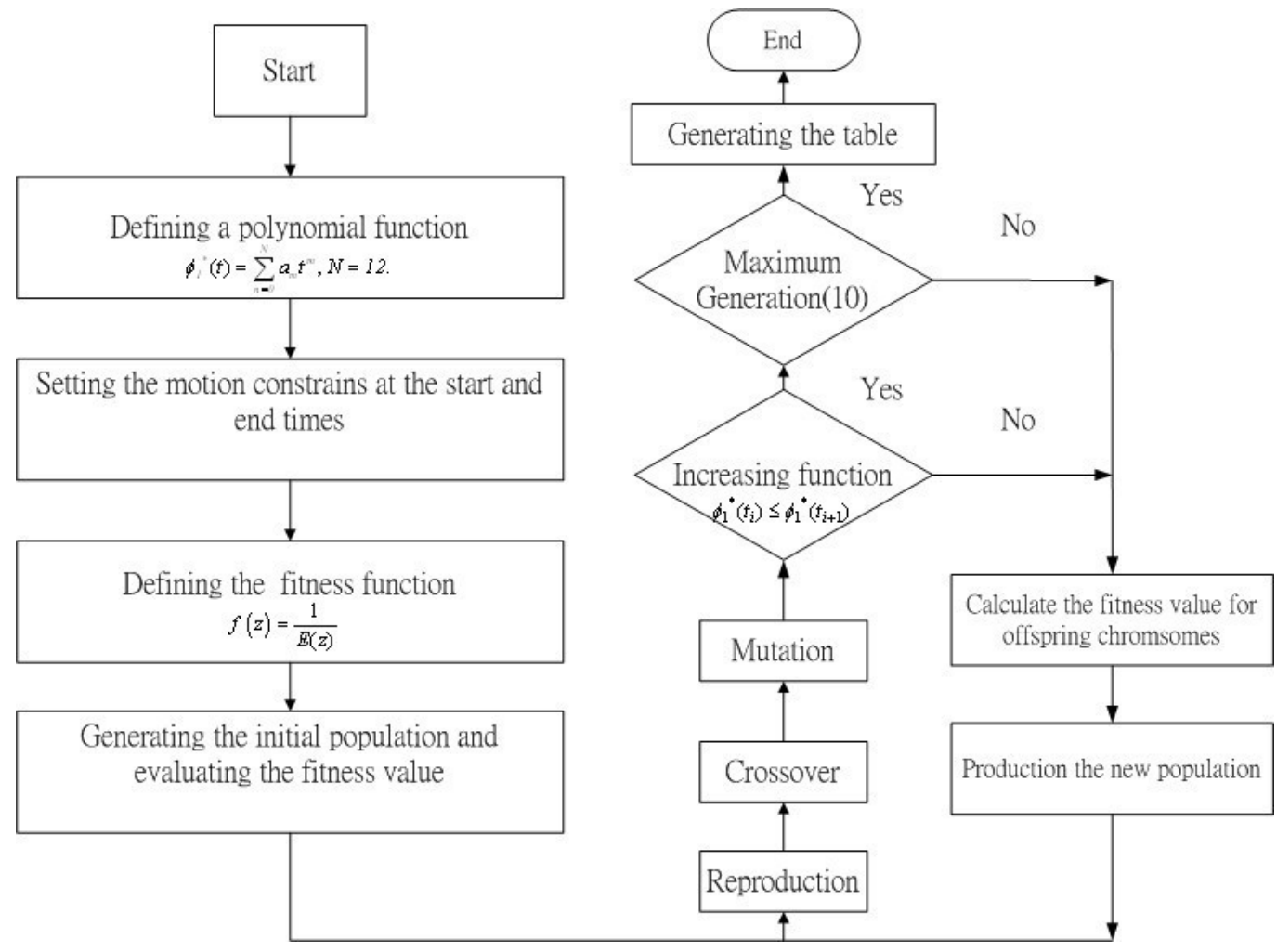

Figure 2 Flow chart of the RGA procedure

for a steady state, as $\dot{\phi}_{1}$ is constant. The function $g\left(\dot{\phi}_{1}\right)$ is positive and depends on many factors such as material properties, lubrication and temperature. It need not be symmetrical, and direction dependent behavior can therefore be captured. For a typical bearing friction, $g\left(\dot{\phi}_{1}\right)$ will monotonically decrease from $g(0)$ when $\dot{\phi}_{1}$ increases. A parameterization of $g\left(\dot{\phi}_{1}\right)$ has been proposed to describe the Stribeck effect as

$$
\sigma_{0} g\left(\dot{\phi}_{1}\right)=\tau_{c}+\left(\tau_{s}-\tau_{c}\right) e^{-\left(\frac{\dot{\phi}_{1}}{\dot{\phi}_{s}}\right)^{2}}
$$

where $\tau_{c}$ is the Coulomb frictional torque, $\tau_{s}$ is the stiction torque and $\dot{\phi}_{s}$ is the Stribeck angular velocity. It follows from (25), (27) and (28) that for the steadystate motion the relation between angular velocity and frictional torque can be given by

$$
\begin{aligned}
\tau_{f} & =\sigma_{0} g\left(\dot{\phi}_{1}\right) \operatorname{sign}\left(\dot{\phi}_{1}\right)+\sigma_{2} \dot{\phi}_{1} \\
& =\tau_{c} \operatorname{sign}\left(\dot{\phi}_{1}\right)+\left(\tau_{s}-\tau_{c}\right) e^{-\left(\frac{\dot{\phi}_{1}}{\dot{\phi}_{s}}\right)^{2}} \operatorname{sign}\left(\dot{\phi}_{1}\right) \\
& +\sigma_{2} \dot{\phi}_{1}
\end{aligned}
$$

Dynamic Equation (2) can be rewritten by adding the frictional torque (29) as

$$
\begin{aligned}
& {\left[J_{0}-J_{1} \cos \left(n \phi_{1}\right)+J_{m}\right] \ddot{\phi_{1}}+B_{m} \dot{\phi_{1}}} \\
& +\mu \sin \left(n \phi_{1}\right) \dot{\phi}_{1}^{2}=K_{t} i_{q}-\tau_{f}(30)
\end{aligned}
$$

\subsection{Sliding function}

Combining the electrical equation (1) for the PMSM, and the dynamic equation (2) for the mechanical system, and using the state variable $x=\left[\begin{array}{lll}\phi_{1} & \dot{\phi}_{1} & i_{q}\end{array}\right]^{T}$, we obtain the dynamic state equation for the LCD glasshandling robot as follows

$$
\dot{\mathbf{x}}=\mathbf{A} \mathbf{x}+\mathbf{B u}+\mathbf{D}
$$

where $A=\left[\begin{array}{ccc}0 & 1 & 0 \\ 0 & \frac{-B_{m}-\mu \sin \left(n \phi_{1}\right) \dot{\phi}_{1}}{J_{0}-J_{1} \cos \left(n \phi_{1}\right)+J_{m}} & \frac{K_{t}}{J_{0}-J_{1} \cos \left(n \phi_{1}\right)+J_{m}} \\ 0 & -\frac{p \lambda_{d}}{L_{q}} & -\frac{R_{s}}{L_{q}}\end{array}\right]$, $B=\left[\begin{array}{c}0 \\ 0 \\ \frac{1}{L_{q}}\end{array}\right], D=\left[\begin{array}{c}0 \\ -\frac{\tau_{f}}{J_{0}-J_{1} \cos \left(n \phi_{1}\right) J_{m}} \\ 0\end{array}\right], u=v_{q}$

To design a VSC [14], we decompose (31) as

$$
\dot{x}=\left[\begin{array}{c}
\dot{x}_{1} \\
\dot{x}_{2}
\end{array}\right]=\left[\begin{array}{ll}
A_{11} & A_{12} \\
A_{21} & A_{22}
\end{array}\right]\left[\begin{array}{l}
x_{1} \\
x_{2}
\end{array}\right]+\left[\begin{array}{l}
B_{1} \\
B_{2}
\end{array}\right] u
$$

where $x_{1}=\left[\begin{array}{c}\phi_{1} \\ \dot{\phi}_{1}\end{array}\right], x_{2}=i_{q}$,

$$
\begin{aligned}
& A_{11}=\left[\begin{array}{ll}
0 & 1 \\
0 & \frac{-B_{m}-u \sin \left(n \phi_{1}\right) \dot{\phi}_{1}}{J_{0}-J_{1} \cos \left(n \phi_{1}\right)+J_{m}}
\end{array}\right], A_{12}=\left[\begin{array}{c}
0 \\
\frac{k_{t}}{J_{0}-J_{1} \cos \left(n \phi_{1}\right)+J_{m}}
\end{array}\right], \\
& A_{21}=\left[\begin{array}{ll}
0 & -\frac{p \lambda_{d}}{L_{q}}
\end{array}\right], A_{22}=-\frac{R_{s}}{L_{q}}, B_{1}=\left[\begin{array}{l}
0 \\
0
\end{array}\right], B_{2}=\frac{1}{L_{q}} .
\end{aligned}
$$


Under the VSC, the system can track the trajectory and approach to the sliding surface, so the sliding surface has to include track object. The sliding function can be expressed as

$$
s=c e=c\left(x-x^{*}\right)=\left[\begin{array}{ll}
c_{1} & c_{2}
\end{array}\right]\left(\left[\begin{array}{l}
x_{1}-x^{*}{ }_{1} \\
x_{2}-x_{2}{ }^{*}
\end{array}\right]\right)
$$

where $c_{1} \in R^{1 \times 2}, c_{2} \in R ; x^{*}{ }_{1}=\left[\begin{array}{c}\phi_{1}{ }^{*} \\ \dot{\phi}_{1}^{*}\end{array}\right], x^{*}{ }_{2}=i^{*}{ }_{q}$

If the system model is on an ideal sliding surface $s=c e=0$, we can determine the equivalent system and an associated linear control input.

In order to consider the linear system, we set $\phi_{1}=0$ in $A_{11}$ and $A_{12}$ of Equation (32). On the sliding surface $s=0$, we can obtain

$$
x_{2}=x_{2}{ }^{*}-\frac{c_{1}}{c_{2}}\left[x_{1}-x_{1}^{*}\right]
$$

Substituting (34) into (32), we have

$$
x^{*}{ }_{1}=A_{11} x_{1}+A_{12}\left(x_{2}{ }^{*}-c_{2}{ }^{-1} c_{1}\left[x_{1}-x_{1}{ }^{*}\right]\right)
$$

Defining $k=c_{2}{ }^{-1} c_{1}$ we rewrite (35) as

$$
x^{*}{ }_{1}=\left(A_{11}-A_{12} k\right) x_{1}+A_{12}\left(x_{2}^{*}+k x_{1}^{*}\right)
$$

It is seen that the locations of poles of the resulting system (36) can be obtained by selecting $k$ and $c_{2}$. Because $\left(A_{11}, A_{12}\right)$ is controllable, we use a pole placement method to select the gain $k$ in (36).

\subsection{Design the VSC law}

The variable structure control [14] can be separated into the equivalent and nonlinear control components as $u=u_{e q}=u_{n}$. The equivalent control can be selected by the following equations

$$
\begin{aligned}
& \dot{x}=A x+B u \\
& \dot{s}=c \dot{e}=0
\end{aligned}
$$

From (37) and (38), we obtain the equivalent control input as

$$
u_{e q}=-(c B)^{-1}\left(c A x-c \dot{x}^{*}\right)
$$

The approaching condition [14] $s \dot{s}<0$ can bring the system model to the sliding surface $s=0$ Selecting the nonlinear control as

$$
u_{e q}=-(c B)^{-1}\left(\alpha_{1} \operatorname{sgn}(s)+\alpha_{2} s\right)
$$

where $\alpha_{1}$ and $\alpha_{2}$ are positive constants. Finally, it follows that

$$
\begin{aligned}
s \dot{s} & =s c\left(A x+B u+D-\dot{x}^{*}\right) \\
& =s c A x-s c B\left((c B)^{-1}\left(c A x-c \dot{x}^{*}\right)\right. \\
& \left.+s c D+(c B)^{-1}\left(\alpha_{1} \operatorname{sgn}(s)+\alpha_{2} s\right)\right)-s c \dot{x}^{*} \\
& =-\alpha_{2} s^{2}-\alpha_{1}|s|+c D s<0, \text { if } \alpha_{1}>|c D|
\end{aligned}
$$

From (41), the approaching condition is negative except on the sliding surface $s=0$.

For suppressing the switch function to create chatter free, we use the saturation function to replace the sign function and write the controller as

$$
\begin{aligned}
u= & -(c B)^{-1}\left(c A x-c x^{*}\right) \\
& -(c B)^{-1}\left(\alpha_{1} \operatorname{sat}(s)+\alpha_{2} s\right)
\end{aligned}
$$

where $\operatorname{sat}(s, \varepsilon)=\left\{\begin{array}{ll}1 & s>\varepsilon \\ s / \varepsilon & |s| \leq \varepsilon \\ -1 & s<-\varepsilon\end{array}= \begin{cases}\operatorname{sign}(s) & |s|>\varepsilon \\ s / \varepsilon & |s| \leq \varepsilon\end{cases}\right.$

\section{Numerical simulation}

\subsection{Trajectory planning}

In numerical simulations, the fourth-order RungeKutta method is employed to calculate the absolute input energy via a windows supported MATLAB software with a sampling time sec and the total time sec. The identified parameters of the LCD glass-handling robot driven by a PMSM are given in Table 1 [8].

The coefficients of the polynomials with 8-12 degrees are obtained by the RGA and are shown in Table 2. In this Table, the feasible domains are taken -1 to 1 for all the coefficients.

Figure 3 (a-h) show the comparisons among 8-12 degrees of polynomials. Figure 3 (a-d) show the designed trajectories of the angular displacements, speeds, accelerations and jerks of Arm 1, respectively. Taking time derivatives of the designed trajectories, we obtain its time derivatives,substitute into (2), and obtain the input current as shown in Figure 3 (e). By using (21), we can obtain input voltage of the PMSM as shown in Figure 3 (f). Figure 3(g, h) show the input energies and the fitness values, respectively. From Figure 3(h), it is seen that the fitness value increases with increasing the degrees' number of polynomials, and all the genes of the chromosome almost converge before the first 10 generations.

From Table2, it can be found that the minimum absolute input energy occurs for the twelve-degree polynomial. This value is lower $-2.574 \%$ than that of eightdegree polynomial. 


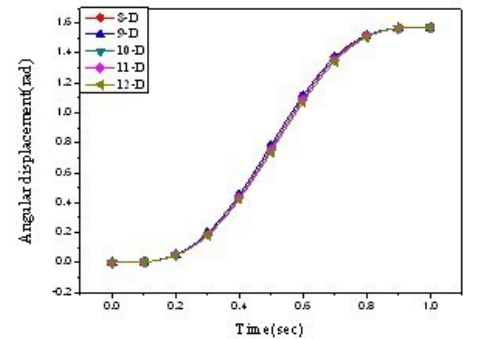

(a)

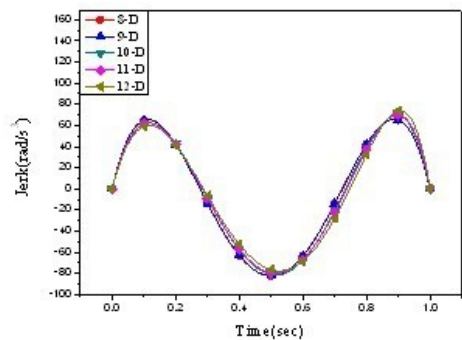

(d)

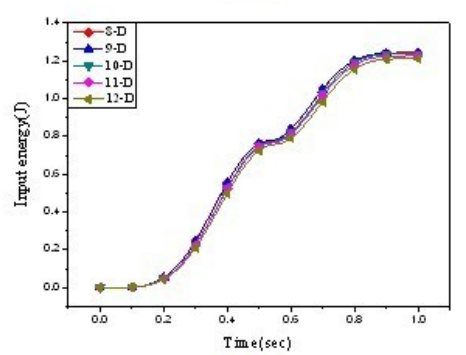

(g)

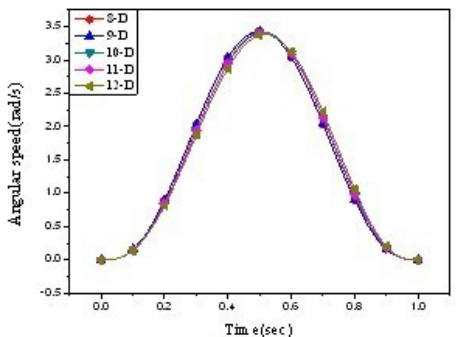

(b)

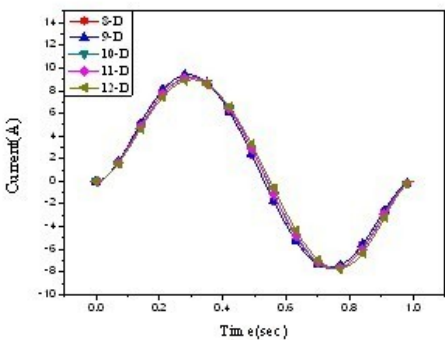

(e)

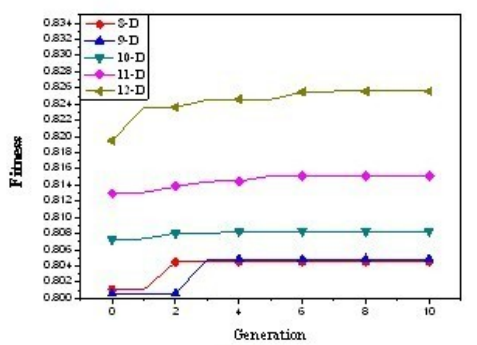

(h)

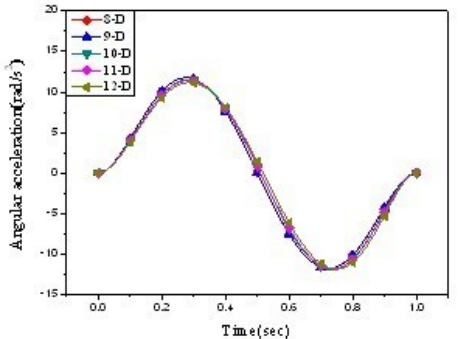

(c)

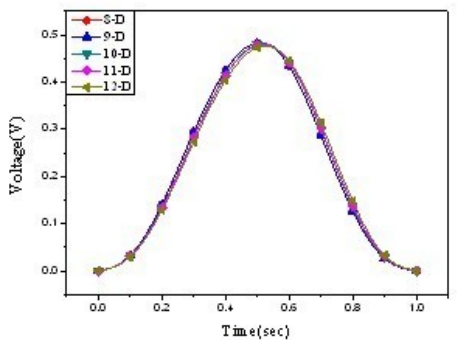

(f)

Figure 3 Comparisons among eight- to twelve-degree polynomials in numerical simulations. (a) The angular displacement. (b) The angular speed. (c) The angular acceleration. (d) The angular jerk. (e) The current. (f) The voltage. (g) The input energy. (h) The fitness functions of evolution history.

Table 2 Coefficients of polynomials and total input energy

\begin{tabular}{|c|c|c|c|c|c|c|c|}
\hline $\begin{array}{c}\text { Polynomial } \\
\text { function } \\
\text { of degree }\end{array}$ & $a_{8}$ & $a_{9}$ & $a_{10}$ & $a_{11}$ & $a_{12}$ & energy $(\mathrm{J})$ & \\
\hline 8 & 0.998 & - & - & - & - & 1.243 & - \\
\hline 9 & 0.988 & -0.740 & - & - & - & 1.242 & -0.080 \\
\hline 10 & 0.836 & 0.941 & -0.926 & - & - & 1.238 & -0.402 \\
\hline 11 & 0.947 & 0.947 & 0.793 & -0.894 & - & 1.228 & -1.207 \\
\hline 12 & 0.763 & 0.836 & 0.810 & 0.978 & -0.977 & 1.211 & -2.574 \\
\hline
\end{tabular}




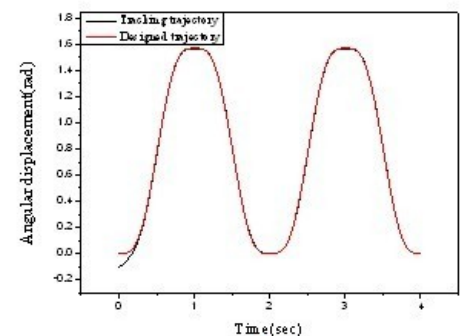

(a)

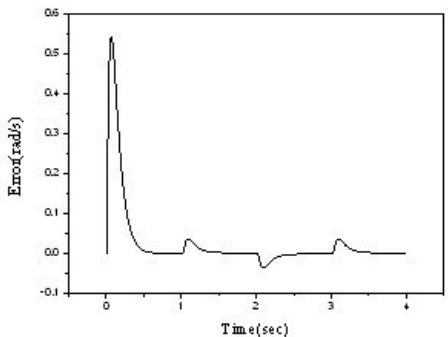

(d)

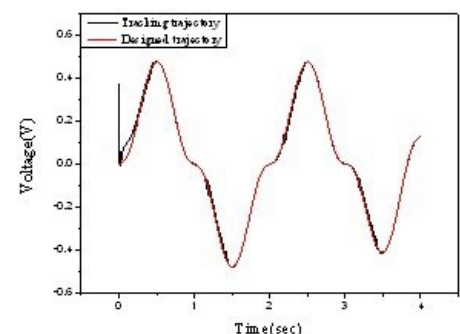

(g)

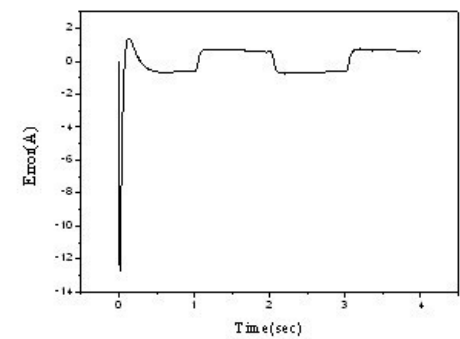

(j)

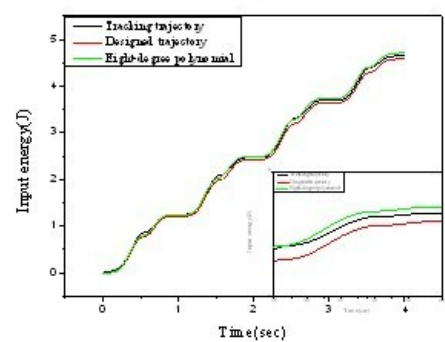

(m)

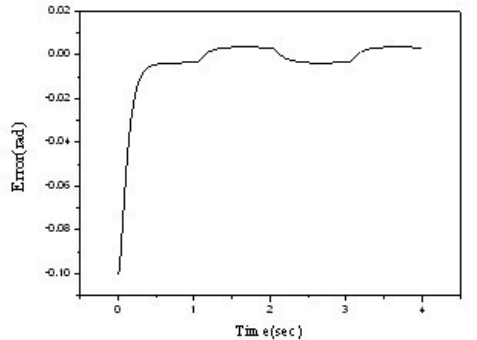

(b)

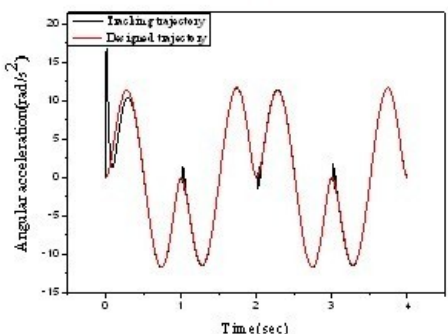

(e)

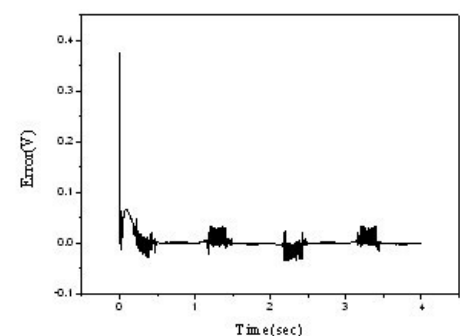

(h)

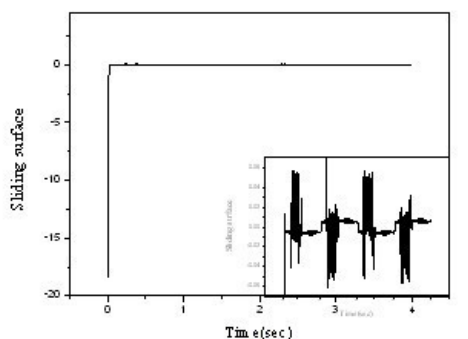

(k)

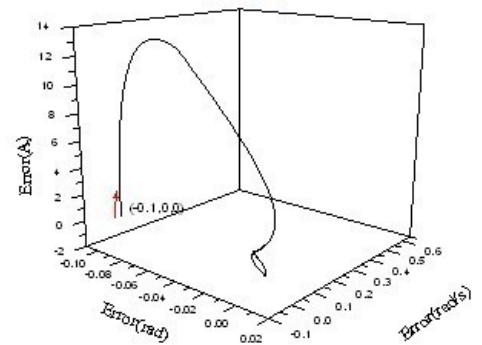

(n)

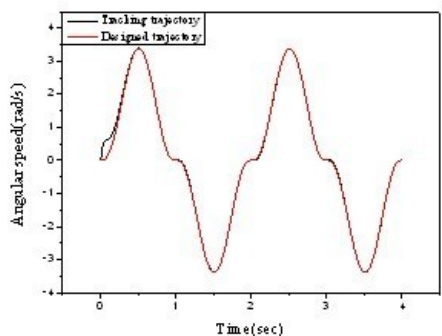

(c)

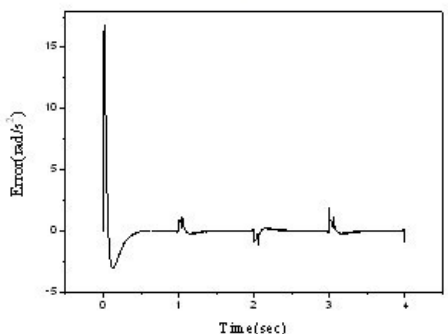

(f)

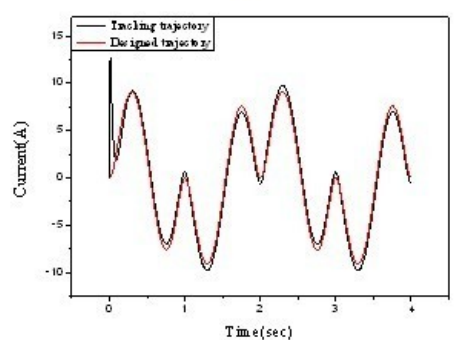

(i)

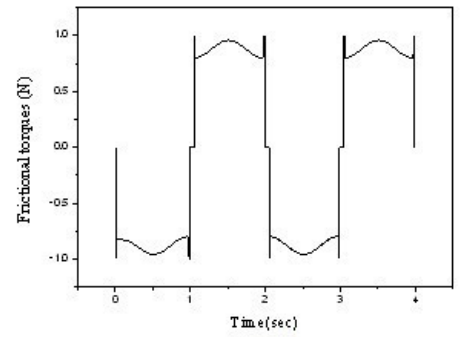

(1)

Figure 4 Comparison among the designed and tracking trajectories. (a) The angular displacement. (b) The errors of angular displacements. (c) The angular speed. (d) The errors of angular speeds. (e) The angular acceleration. (f) The errors of angular accelerations. (g) The voltages. (h) The errors of voltages. (i) The currents. (j) The errors of currents. (k) The sliding surface. (l) The frictional torques of LuGre model. (m) The input energies. (n) The phase trajectory in the state space. 
Table 1 The identified parameters of the robot system

\begin{tabular}{|c|c|}
\hline Parameter & Values \\
\hline$J_{0}\left(k g-m^{2}\right)$ & 0.3585 \\
\hline$J_{1}\left(k g-m^{2}\right)$ & 0.0006708 \\
\hline$u\left(k g-m^{2}\right)$ & 0.0006708 \\
\hline$L_{q}(H)$ & 0.00153 \\
\hline$\lambda_{d}$ & 0.03572 \\
\hline$R_{s}(\Omega)$ & 0.00103 \\
\hline$n$ & 1.9135 \\
\hline$K_{t}(N m / A)$ & 0.55624 \\
\hline$J_{m}\left(k g-m^{2}\right)$ & 0.0679 \\
\hline$B_{m}(\mathrm{Nms} / \mathrm{rad})$ & 0.2858 \\
\hline
\end{tabular}

\subsection{Tracking control}

In numerical simulations of the VSC, the fourthorder Runge-Kutta method is also employed with MATLAB software. The LuGre model parameters and control gains are shown in Table 3 . In order to make system not on the sliding surface at $t=0$, we set $\phi_{1}=$ -0.1 at the start time. To compare the control performance, we show two work cycles with each cycle 2 second. Here, we just show the case with twelvedegree polynomial.

Figures 4 (a-n) compare the designed and tracking trajectories. Figures 4 (a, c, e) compare the angular displacements, speeds and accelerations, respectively, for the designed and tracking trajectories. Figures 4 (b, d, f) show the errors of the angular displacements, speeds, accelerations, and tracking errors, respectively, between the designed and tracking trajectories. Figures $4(\mathrm{~g}, \mathrm{i})$ compare the input voltages and currents, respectively, for the designed and tracking trajectories. Figures $4(\mathrm{~h}, \mathrm{j})$ show the errors of the input voltages and currents, respectively, between the designed and tracking trajectories. Figure $4(\mathrm{k})$ shows the sliding surface. Figure 4 (l) shows the frictional torque of LuGre model. Figure 4 (m) compares the input energies among the designed, tracking and eight-degree polynomial trajectories. It is found that they are very close, and the VSC is proven to track the trajectories well with frictional torques. Figure 4 (n) shows phase trajectory in the state space $\left(\phi_{1}, \dot{\phi}_{1}, \dot{i_{q}}\right)$.

\section{Conclusion}

In this paper, the minimum-energy-based PTP trajectory planning method is performed for an LCD glasshandling robot by utilizing the absolute input energy as the fitness function to determine the polynomial coefficients by the RGA. From the comparisons in nu-
Table 3 The identified parameters of the robot system

\begin{tabular}{|c|c|}
\hline Coulomb frictional torque $F_{c}$ & $0.8(\mathrm{Nm})$ \\
\hline Static frictional torque $F_{s}$ & $1(\mathrm{Nm})$ \\
\hline Stribeck velocity $\dot{\phi}_{s}$ & $0.005(\mathrm{rad} / \mathrm{sec})$ \\
\hline Viscous coefficient $\sigma_{2}$ & 0.047 \\
\hline$c_{1}$ & $(149.2,20.91)$ \\
\hline$c_{2}$ & 1 \\
\hline$\alpha_{1}$ & 150 \\
\hline$\alpha_{2}$ & 110 \\
\hline
\end{tabular}

merical simulations, the minimum-energy input occurs when the highest-degree polynomial is chosen. The nonlinear controller VSC successfully tracks the trajectories and compensates the frictional torques well. Moreover, the proposed methodology in this paper can also be applied to any mechatronic system driven by a PMSM for designing the minimum-energy PTP trajectory using a high-degree polynomial function.

\section{Acknowledgment}

The financial support from the National Science Council of the Republic of China with contract number NSC-97-2221-E-327-016-MY3 is gratefully acknowledged.

\section{References}

[1] X. F. Zha, "Optimal Pose Trajectory Planning for obot Manipulators," Mechanism and Machine Theory, Vol.37, pp.1063-1086, 2002.

[2] F. Valero, V. Mata and A. Besa, "Trajectory Planning in Workspaces with Obstacles Taking into Account the Dynamic Robot Behaviour,' Mechanism and Machine Theory, Vol. 41, pp.525-536, 2006.

[3] A. Gasparetto and V. Zanotto, "A New Method for Smooth Trajectory Planning of Robot Manipulators," Mechanism and Machine Theory, Vol.42, pp.455471, 2007.

[4] L. Biagiotti, "Trajectory planning for automatic machines and robots", Springer Berlin Heidelberg, 2008.

[5] M. S. Huang, Y. L. Hsu and R. F. Fung, "MinimumEnergy System Point-to-Point Trajectory Planning for a Motor-Toggle Servomechanism," accepted by IEEE/ASME Transactions on Mechatronics.

[6] K. F. Man, K. S. Tang and S. K. Wong, "Genetic Algorithms: Concepts and Applications," IEEE, Transactions on Industrial Electronics 43, Vol.5, pp.519534, 1996.

[7] L. Tian and C. Collins, "An Effective Robot Trajectory Planning Method using a Genetic Algorithm," Mechatronics, Vol.14, pp.455-470, 2004. 
[8] R. F. Fung, Y. S. Kung and G. C. Wu, "Dynamic Analysis and System Identification of an LCD Glasshandling Robot Driven by a PMSM," Applied Mathematical Modelling, Vol.34, pp.1360-1381, 2010.

[9] R. F. Fung and Sin-Syun You, "System Identification and Tracking Control for a 3-DOF LCD Glasshandling Robot," International Journal of Automation Smart Technology, Vol.1, No.1, pp.53-66, 2011.

[10] W. Leonnard, "Microcomputer Control of High Dynamic Performance AC Driver - a surver," Automatica, Vol.22, pp.1-19, 1986.

[11] Canudas de Wit, C., Olsson, H., Aström, K., and Lishinsky, P., "A New Model for Control of Systems with Friction," IEEE Trans. Autom. Control, 40, pp.419-425, 1995.

[12] J. L. Ha, R. F. Fung, C. F. Han, J. R. Chang, "Effects of Frictional Models on the Dynamic Response of the Impact Drive Mechanism, "Transactions of the ASME, Vol.128, pp.88-96, 2006.

[13] A. Damiano, G. L. Gatto, I. Marongiu and A. Pisano, "Second-Order Sliding- Mode Control of DC Drives," IEEE, Transactions on Industrial Electronics, Vol.54, pp.364-373, 2007.

[14] J. H. Lee, P. E. Allaire, G. Tao and X. Zhang, "Integral Sliding-Mode Control of a Magnetically Suspended Balance Bean: Analysis, Simulation, and Experiment," IEEE/ASME Transactions on Mechatronics, Vol.6, pp.338-346, 2001. 